\title{
A REALIDADE AUMENTADA EM SMARTPHONES NA EXPLORAÇÃO DE INFORMAÇÕES ESTATÍSICAS E CARTOGRÁFICAS
}

\author{
Augmented Reality in smartphones for cartographic information exploration \\ FABIANA SILVA PINTO ${ }^{1}$ \\ JORGE ANTONIO SILVA CENTENO ${ }^{2}$ \\ ${ }^{1}$ IBGE - Instituto Brasileiro de Geografia e Estatística \\ Unidade Estadual do Rio Grande do Sul \\ fabiana.spinto@gmail.com \\ ${ }^{2}$ UFPR - Universidade Federal do Paraná \\ Curso de Pós-Graduação em Ciências Geodésicas \\ centeno@ufpr.br
}

\begin{abstract}
RESUMO
A Realidade Aumentada é uma nova forma de interação homem-máquina, que permite aos usuários examinar a realidade mediante a visualização/inclusão de informação adicional, previamente selecionada, relevante para a solução de um determinado problema, em tempo real junto com a realidade percebida pelo ser humano. Basicamente, uma camera de vídeo proporciona cenas em tempo real e um sistema de computador adiciona à imagem real a informação virtual, como por exemplo a inserção de nomes de elementos geográficos como rios e montanhas os quais não são visíveis a olho nú e podem ser inseridos como informação virtual sobre à imagem real. O objetivo deste trabalho é explorar o uso da realidade aumentada em smartphones na visualização e representação de dados do Censo e Banco de Nomes Geográficos do Brasil, logo informações estatísticas e cartográficas respectivamente, ambos disponibilizados pelo Instituto Brasileiro de Geografia e Estatística - IBGE, bem como validar a representação dos dados através do uso de diferentes variáveis visuais. Para alcançar o objetivo proposto um módulo de realidade aumentada para smartphone foi desenvolvido, o qual utiliza diferentes variáveis visuais para a representação dos dados. A presente pesquisa visa permitir aos estudantes, técnicos e usuários de cartográfia a obter, explorar, solucionar problemas, facilitar a compreensão e interpretação de informações cartográficas e
\end{abstract}


estatísticas em tempo real através de uma nova forma de visualização: a realidade aumentada.

Palavras-chave: Realidade Aumentada; Smartphone; Sensores; Variáveis Visuais; dados do IBGE.

\section{ABSTRACT}

Augmented Reality is a new form of human-machine interaction which allows users to look the reality through the visualization / inclusion of virtual information, previously selected, relevant for solving a given problem, in real time together with the real world. Basically, a video camera provides scenes in real time and a computer system, enhances the scene by adding virtual information to the image. Basically, a video camera provides real-time scenes and a computer system adds to the real image virtual information, as for example, the inclusion of names of geographic features like rivers and mountains which are not visible directly to the eye and can be visualized as virtual information together with the real world. The aim of this paper is to explore the use of augmented reality on smartphones in the view of the representation of Census data and Bank of Geographical Names in Brazil, both provided by the Brazilian Institute of Geography and Statistics - IBGE, as well as validating the data representation by the use of different visual variables. To achieve the objective proposed a module of augmented reality for smartphone was developed, which uses different variables for the visual representation of data. This research hopes to enable students, technicians and mapping users to obtain and explore the information data from IBGE through a new way of viewing: augmented reality.

Keywords: Augmented Reality; Smartphones; Sensors; Visual Variables; IBGE Data.

\section{INTRODUÇÃO}

Recentemente, os smartphones têm se apresentado como uma plataforma multifuncional com sensores integrados, tais como microfones, tela colorida e sensível ao toque, camera, acelerômetro, magnetômetro, detector de luminosidade, dentre outros. Devido a multifuncionalidade dos sensores, os smartphones estão além do simples objetivo de proporcionar a comunicação entre as pessoas. De acordo com o GARTNER GROUP (2011) mais de 1,5 bilhão de unidades de telefones portáteis (celulares) serão vendidos em 2012, dos quais 800 milhões serão smartphones. Em apenas três anos, segundo análise da consultoria International Data Corporation (IDC), o mercado mundial de smartphones cresceu 146\%, passando de 124 milhões de unidades em 2007 para 305 milhões em 2010.

Consequentemente, o desenvolvimento de aplicações voltadas para este segmento também está em evolução. Dentre os aplicativos disponíveis para smartphones podem ser destacados os que permitem novas formas de busca de informações com o uso de sistemas de realidade aumentada (SRA) como o Layar e 
o Wikitude. A realidade aumentada é definida segundo Milgram E Kishino, (1994) como a combinação de cenas do mundo real com informações virtuais. Os smartphones têm se apresentado como uma plataforma inovadora para um SRA, pois são mais leves e portáteis quando comparados aos sistemas tradicionais de realidade aumentada como apresentado em Sutherland (1968) que necessitam de GPS, sistema inercial, óculos especiais e computador para o seu funcionamento. $\mathrm{Na}$ literatura são apresentadas experiências sobre o uso da realidade aumentada em smartphones no ensino escolar (MAQABLEH \& SIDHU, 2010), na descrição de pontos turísticos (MARIMON, et. al.; 2010), como guia em museus (MIYASHITA, 2008), na visualização de informações sobre o mapeamento da Grã-Bretanha (RADBURN, 2006), na visualização de informações pessoais através de cartão de visitas (WENG, et. al., 2011). Embora a RA encontre aplicações alguns do sentidos humanos (AZUMA, 2001), neste estudo apenas a aplicação à percepção visual é abordada.

Tendo em vista as diferentes aplicações que existem atualmente em realidade aumentada o objetivo geral deste trabalho é explorar o uso da realidade aumentada em smartphones na visualização e representação de dados do Censo e Banco de Nomes Geográficos do Brasil, ambos disponibilizados pelo Instituto Brasileiro de Geografia e Estatística - IBGE. Como objetivos específicos estão o desenvolvimento de um módulo de realidade aumentada para smartphones para a visualização dos dados como pontos de interesse e a validação da representação adotada para os dados através do uso das variáveis visuais: forma, cor e tamanho.

A motivação da presente pesquisa é que a realidade aumentada venha a auxiliar e solucionar problemas na visualização dos dados do Censo e Banco de Nomes Geográficos do Brasil em trabalhos de campo realizados pelo IBGE, bem como a de permitir aos estudantes e usuários de cartográfia a obter e explorar as informações estatísticas e cartográficas produzidas pelo IBGE através de uma nova forma de visualização: a realidade aumentada. $\mathrm{O}$ uso da realidade aumentada em trabalhos e pesquisas de campo pode reduzir o esforço mental do usuário necessário para interpretar as informações do Censo e Banco de Nomes Geográficos do Brasil que atualmente são apresentados em forma de tabelas, gráficos, textos e mapas digitais ou impressos, considerando que os usuários nem sempre possuem o conhecimento prévio da região e nem experiência para realizar a interpretação das informações. Desta forma a consulta às informações ocorre de forma indireta pois o sistema de realidade aumentada se encarrega de buscar e disponibilizar para o usuário. Outro problema que pode ser minizado é o tempo gasto em campo para a localização de pontos de interesse que através da realidade aumentada pode reduzido, devido a consulta e visualização em tempo real dos pontos em campo.

\section{MATERIAIS}

Um sistema de Realidade Aumentada depende da interação entre diferentes sensores para captura de imagens, estimativa de posição e atitude, bem como para a visualização dos dados. As melhorias em termos resolução e tamanho de tela 
disponíveis em um smartphone oferecem boa qualidade na visualização tanto das informações virtuais quanto da cena real em um sistema de realidade aumentada. Porém, a busca pela portabilidade implicou na redução da precisão da estimativa de posição e atitude devido aos sensores integrados nos smartphones serem elaborados de forma mais simples, para a redução de custos e dimensões do aparelho. Logo, no desenvolvimento de um SRA deve ser verificada se a finalidade da aplicação necessita de alta (milímetros) ou baixa precisão (metros), como por exemplo para localização de dutos de petróleo e a localização de elementos geográficos como rios, respectivamente.

$\mathrm{Na}$ atualidade, existem vários sistemas operacionais (SO) para smartphones. Porém, estes podem ser mutuamente incompatíveis e cada dispositivo móvel suporta apenas um em particular. Portanto, para maximizar o alcance da aplicação foi escolhido um sistema operacional que permitisse o uso em mais de um único modelo e fabricante de smartphone, como mostra a Tabela 1. Esta tabela apresenta as principais caracterísitcias dos smartphones, alguns fabricantes e os sistemas operacionais utilizados.

Tabela 1 - Os smartphones e os sistemas operacionais utilizados.

\begin{tabular}{l|l|l|l|l}
\hline Smartphone & iPhone 4 & $\begin{array}{l}\text { HTC } \\
\text { Incredible }\end{array}$ & $\begin{array}{l}\text { Samsung } \\
\text { Galaxy }\end{array}$ & $\begin{array}{l}\text { Moto } \\
\text { Milestone }\end{array}$ \\
\hline Fabricante & Apple & HTC & Samsung & Motorola \\
\hline $\begin{array}{l}\text { Sistema } \\
\text { Operacional }\end{array}$ & iOS4 & $\begin{array}{l}\text { Android 2.1 } \\
\text { com HTC } \\
\text { Sense }\end{array}$ & Android 2.1 & $\begin{array}{l}\text { Android } \\
2.1\end{array}$ \\
\hline Processador & Apple A4 & $\begin{array}{l}\text { Qualcomm } \\
1 \mathrm{GHz}\end{array}$ & $\begin{array}{l}\text { ARM } \\
\text { Cortex A8 } \\
1 \mathrm{GHz}\end{array}$ & $\begin{array}{l}\text { ARM } \\
\text { Cortex A8 } \\
550 \mathrm{GHz}\end{array}$ \\
\hline $\begin{array}{l}\text { Armazena- } \\
\text { mento }\end{array}$ & $16 \mathrm{~Gb} / 32 \mathrm{~Gb}$ & $8 \mathrm{~Gb}$ & $8 \mathrm{~GB} / 16 \mathrm{~Gb}$ & $550 \mathrm{Mb}$ \\
\hline $\begin{array}{l}\text { Armazena- } \\
\text { mento } \\
\text { expansível }\end{array}$ & não & $\begin{array}{l}\text { microSD, } \\
2 \mathrm{~Gb} \text { incluso }\end{array}$ & microSD & $\begin{array}{l}\text { microSD, } \\
8 \mathrm{~Gb} \\
\text { incluso }\end{array}$ \\
\hline $\begin{array}{l}\text { Duração } \\
\text { Bateria }\end{array}$ & 7 horas & 5.2 horas & 6.5 horas & 4.5 horas \\
\hline $\begin{array}{l}\text { Código } \\
\text { Aberto }\end{array}$ & não & \multicolumn{4}{|c}{ sim } \\
\hline $\begin{array}{l}\text { Loja } \\
\text { aplicativos }\end{array}$ & Apple Store & \multicolumn{4}{|l}{} \\
\hline
\end{tabular}

Também foi analisada a tendência do mesmo SO estar presente em outros dispositivos móveis como tablets ou netbook, os quais apresentam grande crescimento no mercado e podem ser utilizados no futuro com a aplicação de RA. A 
Tabela 2 apresenta as principais caracterísitcas de alguns tablets, seus fabricantes e sistema operacional adotado.

Tabela 2 - Fabricantes de tablets e os sistemas operacionais utilizados.

\begin{tabular}{l|l|l|l|l}
\hline Tablet & Prime & IPad 2 & Kindle Fire & $\begin{array}{l}\text { Nook } \\
\text { Tablet }\end{array}$ \\
\hline Fabricante & Asus & Apple & Amazon & $\begin{array}{l}\text { Barnes \& } \\
\text { Noble }\end{array}$ \\
\hline $\begin{array}{l}\text { Sistema } \\
\text { Operacional }\end{array}$ & $\begin{array}{l}\text { Android } \\
3.2\end{array}$ & iOS5 & $\begin{array}{l}\text { Android } \\
\text { customizado }\end{array}$ & $\begin{array}{l}\text { Android } \\
\text { customizado }\end{array}$ \\
\hline $\begin{array}{l}\text { Armazena- } \\
\text { mento }\end{array}$ & $32 \mathrm{~Gb} / 64 \mathrm{~Gb}$ & $\begin{array}{l}16 \mathrm{~Gb} / 32 \mathrm{~Gb} \\
164 \mathrm{~Gb}\end{array}$ & $8 \mathrm{~Gb}$ & $16 \mathrm{~Gb}$ \\
\hline $\begin{array}{l}\text { Armzenamen- } \\
\text { to expansível }\end{array}$ & microSD & não & não & microSD \\
\hline $\begin{array}{l}\text { Duração da } \\
\text { Baterial }\end{array}$ & 12 horas & 10 horas & 7,5 horas & 9 horas \\
\hline $\begin{array}{l}\text { Loja de } \\
\text { aplicativos }\end{array}$ & $\begin{array}{l}\text { Android } \\
\text { Market }\end{array}$ & $\begin{array}{l}\text { Apple } \\
\text { Store }\end{array}$ & \multicolumn{3}{|c}{ Android Market } \\
\hline
\end{tabular}

A partir dos critérios apresentados e de trabalhos acadêmicos que também utilizam o Android como SO, como pode ser encontrado em DOMHAN (2010) e GASSMANN (2010), o SO Android foi escolhido para o uso nesta pesquisa, em sua versão 2.2 denominada Froyo. O Android é baseado em Linux e é uma das primeiras plataformas para aplicações móveis, livre e com código aberto administrado pela especialista em web Google e a Open Handset Alliance. O Android Software Development Kit (SDK) fornece as ferramentas, emulador e as bibliotecas necessárias para inicar o desenvolvimento de aplicações em dispositivos Android. O módulo de realidade aumentada foi desenvolvido a partir de um framework de código aberto para realidae aumentada denominado mix ar e disponível sob a licença GNU GPLv3 (General Public License versão 3).

\subsection{Hardware}

Para este trabalho foi escolhido o smartphone Samsung 19000 Galaxy S. Uma das motivações para a escolha deste dispositivo foi devido a utilização do sistema operacional Android, bem como as caracterísitcas dos sensores que integram o dispositivo. As características básicas deste smartphone são: Display Super AMOLED com 800 x 480 pixel; Tela multi-toque capacitativa; Sistema operacional Android 2.2.1; CPU ARM Cortex A8 com 1 GHz; 512 MB RAM; Memória de 16 GB de armazenamento; Câmera com 5 megapixel; Acelerômetro com 3-eixos; Magnetômetro com 3-eixos; aGPS (assisted GPS), e Conexão a Internet $3 \mathrm{G}$ e WiFi. A duração da bateria pode variar em função dos aplicativos que estão instalados e que utilizam conexão a Internet para a atualização de seus dados como emails, redes 
sociais, dentre outros. Se o aparelho for utilizado apenas utilizado para ligações a bateria pode durar cerca de 7 horas, em aplicações de realidade aumentada este número baixa para em média 4 horas. Dentre os sensores presentes no smartphone para a aplicação em RA podem ser destacados: a câmera, aGPS, acelerômetro e magnetômetro.

\subsection{Software}

O SO Android é formado por dois importantes componentes: o sistema Linux que é a base para o sistema operacional e o Java que é a linguagem para o desenvolvimento. O Eclipse foi selecionado como ambiente de desenvolvimento por ser compatível com o Android ADT (Android Development Tools), necessário para compilações específicas em dispositivos móveis. Para aplicações em Android é necessária a utilização do Android SDK (Software Development Kit) o qual possui as bibliotecas necessárias para a manipulação dos sensores e informações presentes em um smartphone (MEIER, 2010).

O módulo do SRA desenvolvido também conta com páginas web para fornecer um maior detalhamento das informações virtuais a serem visualizadas. Para isso foi utilizada a linguagem HTML (HyperText Markup Language). Esta linguagem é utilizada em páginas web devido a sua capacidade de formatação e diagramação de hipertexto/hipermídia e é interpretada por todos os navegadores disponíveis.

\subsection{Dados}

As informações utilizadas pelo SRA como elementos virtuais são os dados do Censo e Nomes Geográficos do Brasil, ambos produzidos pelo IBGE que serão a seguir detalhados. O IBGE na produção destes dados realiza pesquisas que envolvem um extenso trabalho de campo e seus resultados podem ser utilizados para diversas finalidades. Os Censos demográficos, por exemplo, produzem informações para a definição de políticas públicas e a tomada de decisões de investimentos e constituem a única fonte de referência sobre a situação de vida da população nos municípios e em seus recortes internos, como setores, distritos, bairros e localidades. O nome geográfico, segundo IBGE (2011), é um topônimo padronizado acrescido de atributos que o caracteriza como um conjunto etnográfico, etimológico e histórico, referenciado geograficamente e inserido em contexto temporal. $\mathrm{O}$ topônimo é concebido como a denominação de acidentes naturais e culturais que são representados em documentos cartográficos, em diversas escalas. Os nomes geográficos são uma relevante marca cultural porque, além de refletir seus padrões de ocupação e sua diversidade linguística, caracterizam a nomenclatura associada aos acidentes geográficos. Desde 2010 foi implantado pelo IBGE o Comitê de Nomes Geográficos do Brasil, o qual é responsável pelo estabelecimento das normas de padronização dos nomes geográficos brasileiros em documentos cartográficos. O nome geográfico é composto por duas partes: a primeira referida ao termo genérico como sendo a denominação genérica do tipo de acidente geográfico 
natural ou construído pelo homem; e a segunda referida ao termo específico como sendo a denominação específica que singulariza a identidade do elemento geográfico. Exemplo: Rio São Francisco; termo genérico: Rio; termo específico: São Francisco.

Os dados foram divididos em duas categorias: Nome Geográfico do Brasil e Censo. Estes fornecem uma grande quantidade de elementos e devido ao seu grande volume apenas alguns dos elementos que os compõem foram selecionados para a visualização no SRA como mostra a Tabela 3.

Tabela 3 - Dados selecionados.

\begin{tabular}{l|l|l}
\hline \multirow{2}{*}{ Dados } & $\begin{array}{l}\text { Nome Geográfico do } \\
\text { Brasil }\end{array}$ & Censo \\
\hline \multirow{4}{*}{} & Parque Municipal & $\mathrm{N}^{\circ}$. Setor \\
\cline { 2 - 3 } & Parque Estadual & Número de habitantes \\
\cline { 2 - 3 } & Parque Florestal & Número de homens \\
\cline { 2 - 3 } & Barragem & Número de mulheres \\
\cline { 2 - 3 } & Aeroporto & Número de Crianças \\
\hline & & Renda Média \\
\hline
\end{tabular}

Em relação aos dados do Censo, no SRA apenas a informação referente ao número do setor será visualizado diretamente no SRA. Entende-se como setor, de maneira simplificada, a menor unidade territorial de coleta que pode ser percorrida por um único recenseador. As demais informações sobre o setor são visualizadas a através de páginas da web desenvolvidas especificamente para o SRA que apresentam as demais informações: $\mathrm{n}$. de habitantes, n. de homens, n. de mulheres, número de crianças, renda média dos últimos dois Censos. Foi necessário esta diferenciação a fim de evitar a sobreposição de informações na visualização dos elementos no SRA.

\section{MÉTODO}

Na elaboração de sistema de realidade aumentada é necessário determinar a posição e atitude do smartphone em tempo real, bem como são necessárias uma série de transformações entre os sistemas de coordenadas envolvidos no processo, a fim de unir em uma única imagem a cena real e os elementos virtuais. Sendo assim, o módulo de realidade aumentada desenvolvido também seguiu estes critérios. A determinação da posição e as transformações das coordenadas envolvidas são descritas nos itens seguintes.

\subsection{Determinação da posição}

A determinação da posição de um smartphone é baseada em aGPS. O aGPS determina sua posição através das informações disponibilizadas pelas estações bases 
da rede de telefonia celular, com a ajuda de um servidor remoto. A conexão inicial não é feita diretamente com o satélite, mas sim com uma antena de telefonia celular que previamente armazenou a localização destes satélites e as transmite para o smartphone em alta velocidade. Assim o aGPS reduz o tempo que um aparelho com GPS leva para localizar a sua posição atual, conhecido como TTFF (Time To First Fix). É necessário o uso de aGPS, pois a antena usada em smartphones são geralmente menores do que as do GPS, portanto mais simples em sua construção e menos sensíveis.

\subsection{Determinação da atitude}

Para a estimativa da atitude do smartphone são usados acelerômetros e magnetômetros. Os acelerômetros mais simples são dispositivos eletro-mecânicos conhecidos como MEMS (Micro Electro-Mechanical Systems). A aplicação mais encontrada em smartphones para o acelerômetro é a mudança da orientação da tela ao girar o aparelho em relação ao seu eixo. Como o smartphone não possui um giroscópio para a obtenção dos valores de sua atitude (roll, pitch, yaw/azimute), é necessário calcular estes valores com ajuda do acelerômetro e magnetômetro.

Os smartphones para estabelecer a orientação do sistema de coordenadas dos seus eixos adotam o padrão de fábrica denominado NED (North, East, Down). O eixo $\mathrm{x}$ do telefone é a direção Norte a qual a bússola aponta, o eixo y aponta para a direita e o eixo $z$ aponta para baixo, como mostra a Figura 1. O eixo do acelerômetro é representado por Gx, Gy, Gz e o do magnetômetro por Bx, By, Bz. Para obter melhores resultados, o aparelho pode ser inicialmente calibrado, orientado-o para o Norte numa superfície plana.

Figura 1 - Sistema de coordenadas do smartphone.

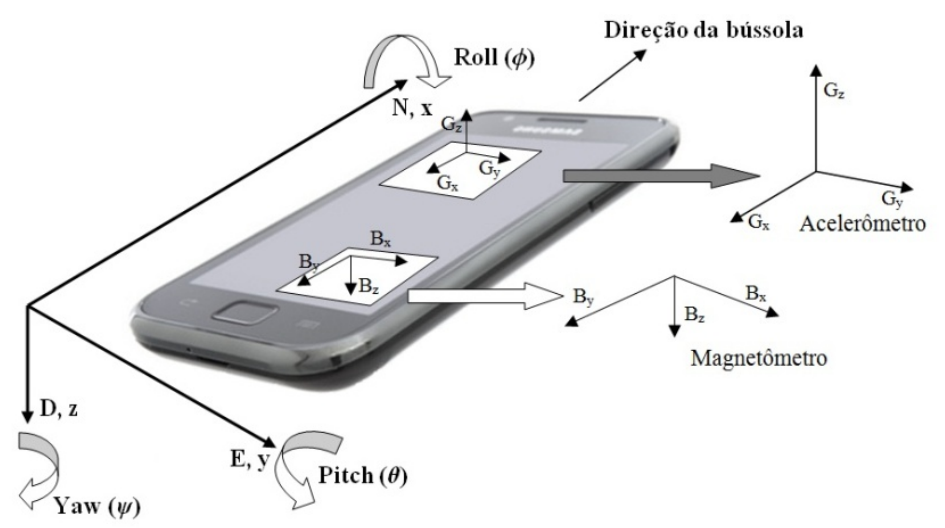

$\mathrm{O}$ aparelho primeiro determina as três componentes da gravidade medidas pelo acelerômetro. $\left[\mathrm{G}_{\mathrm{px}} \mathrm{G}_{\mathrm{py}} \mathrm{G}_{\mathrm{pz}}\right.$ ]' e com base no modelo de transformação para o sistema 
geodésico, aplicando a matriz de rotação mostrada na equação 1 calcula os ângulos roll ( ) e pitch $(\theta)$.

$$
\boldsymbol{R}_{y}(-\theta) \boldsymbol{R}_{x}(-\phi) \boldsymbol{G}_{p}=\boldsymbol{R}_{y}(-\theta) \boldsymbol{R}_{x}(-\phi)\left(\begin{array}{l}
G_{p x} \\
G_{p y} \\
G_{p z}
\end{array}\right)=\boldsymbol{R}_{z}(\psi)\left(\begin{array}{l}
0 \\
0 \\
g
\end{array}\right)=\left(\begin{array}{l}
0 \\
0 \\
g
\end{array}\right)
$$

Expandindo a equação 1, obtém-se a equação 2.

$$
\left(\begin{array}{ccc}
\cos \theta & \sin \theta \sin \phi & \sin \theta \cos \phi \\
0 & \cos \phi & -\sin \phi \\
-\sin \theta & \cos \theta \sin \phi & \cos \theta \cos \phi
\end{array}\right)\left(\begin{array}{l}
G_{p x} \\
G_{p y} \\
G_{p z}
\end{array}\right)=\left(\begin{array}{l}
0 \\
0 \\
g
\end{array}\right)
$$

A componente y da equação 2 define o ângulo roll $(\varphi)$ como:

$$
\begin{gathered}
G_{p y} \cos \phi-G_{p z} \sin \phi=0 \\
\Rightarrow \tan (\phi)=\left(\frac{G_{p y}}{G_{p z}}\right)
\end{gathered}
$$

A componente $\mathrm{x}$ da equação 2 define $\mathrm{o}$ ângulo pitch $(\theta)$ como:

$$
\begin{gathered}
G_{p x} \cos \theta+G_{p y} \sin \theta \sin \phi+G_{p z} \sin \theta \cos \phi=0 \\
\Rightarrow \tan (\theta)=\left(\frac{-G_{p x}}{G_{p y} \sin \phi+G_{p z} \cos \phi}\right)
\end{gathered}
$$

A seguir o aparelho determina as componentes do sensor magnetômetro. [Bfx Bfy Bfz]' e com base no modelo de transformação do sistema do magnetômetro para o sistema geodésico, calcula a componente angular yaw $(\psi)$. O modelo de transformação, neste caso é dado pela equação 5, onde o vetor [Bpx, Bpy, Bpz]' representa as componentes do sensor magnetômetro e o vetor [Vx Vy Vz]' são as componentes que modelam campos magnéticos dispersos. 


$$
=\left(\begin{array}{c}
\left(B_{p x}-V_{x}\right) \cos \theta+\left(B_{p y}-V_{y}\right) \sin \theta \sin \phi+\left(B_{p z}-V_{z}\right) \sin \theta \cos \phi \\
\left(B_{p y}-V_{y}\right) \cos \phi-\left(B_{p z}-V_{z}\right) \sin \phi \\
-\left(B_{p x}-V_{x}\right) \sin \theta+\left(B_{p y}-V_{y}\right) \cos \theta \sin \phi+\left(B_{p z}-V_{z}\right) \cos \theta \cos \phi
\end{array}\right)=\left(\begin{array}{c}
B_{f x} \\
B_{f y} \\
B_{f z}
\end{array}\right)
$$

As componentes x e y da equação 5 fornecem valor do ângulo yaw $(\psi)$ obtido é relativo ao Norte magnético:

$$
\begin{gathered}
\cos \psi B \cos \delta=B_{f x} \\
\sin \psi B \cos \delta=-B_{f y} \\
\Rightarrow \tan (\psi)=\left(\frac{-B_{f y}}{B_{f x}}\right)=\left(\frac{\left(B_{p z}-V_{z}\right) \sin \phi-\left(B_{p y}-V_{y}\right) \cos \phi}{\left(B_{p x}-V_{x}\right) \cos \theta+\left(B_{p y}-V_{y}\right) \sin \theta \sin \phi+\left(B_{p z}-V_{z}\right) \sin \theta \cos \phi}\right)
\end{gathered}
$$

\subsection{Transformação das coordenadas envolvidas no SRA}

Em um sistema de realidade aumentada além de ser necessário conhecer a posição do usuário, neste caso a posição do smartphone, é necessário uma série de transformações entre os sistemas de coordenadas a fim de fundir em uma única imagem a cena real e as informações adicionais, no caso os elementos virtuais. $\mathrm{O}$ mesmo processo de transformações foi desenvolvido no módulo do sistema de realidade aumentada. Logo, a referência geométrica entre a cena real e as informações virtuais descreve uma concatenação de várias transformações entre os diferentes sistemas de coordenadas (GONZALES; WOODS, 2000). No Android tais transformações estão armazenadas dentro de métodos, sendo que cada método corresponde a um dos sensores utilizados no SRA. A partir dos valores fornecidos pelos sensores e disponibilizados pelo Android, as equações de 7 a 12 descritas a seguir são calculadas. A equação 7 define a transformação do sistema de coordenadas do aGPS (XYZ) para o sistema de coordenadas do display da imagem, onde o sobrescrito AeM significa os dados de atitude obtidos do Acelerômetro e Magnetômetro.

$$
T_{W G S 84}^{\text {display }}=S_{C C D}^{\text {display }} \cdot P_{C P}^{C C D} \cdot T_{\text {AeM }}^{C P} \cdot T_{\text {Origem }}^{\text {AeM }} \cdot T_{G P S}^{\text {Origem }}
$$

A partir dos valores dos ângulos de atitude obtidos do acelerômetro e magnetômetro, bem como a posição do usuário/smartphone obtidas a partir do aGPS as respectivas matrizes de transformação são calculadas. Estas definem a seqüência de transformações de coordenadas do mundo real para o mundo virtual que ocorrem no SRA. As transformações T, P, S apresentadas na equação 7 podem ser descritas como segue: 
A equação 8 apresenta a matriz de transformação genérica $\left(T_{\text {antes }}^{\text {depois }}\right)$ que e inclui as rotações $\mathrm{r}$ e as translações $\mathrm{X}, \mathrm{Y}, \mathrm{Z}$ necessárias para a transformação entre os sistemas.

$$
T_{\text {antes }}^{\text {depois }}=\left[\begin{array}{cccc}
r_{11} & r_{12} & r_{13} & X 0 \\
r_{21} & r_{22} & r_{23} & Y 0 \\
r_{31} & r_{32} & r_{33} & Z 0 \\
0 & 0 & 0 & 1
\end{array}\right]_{\text {antes }}^{\text {depois }}
$$

Para transformar as coordenadas tridimensionais do sistema com origem no ponto principal da camera para o plano do CCD é aplicada uma transformação projetiva $\left(P_{C P}^{C C D}\right)$ com a matriz da equação 9 . Um fator de escala na direção das linhas e colunas do chip CCD da camera é aplicado para escalar o tamanho da imagem. Onde tpx, tpy é a dimensão do pixel da camera e $x o$ e yo os valores do ponto principal. Esses parâmetros foram determinados no momento da calibração da camera.

$$
P_{C P}^{c c d}=\left[\begin{array}{ccc}
\frac{1}{t p_{x}} & 0 & x o \\
0 & \frac{1}{t p_{y}} & y o \\
0 & 0 & 1
\end{array}\right]
$$

A transformação entre o chip CCD e a tela display do smartphone $\left(S_{C C D}^{\text {display }}\right)$ é de apenas um fator de escala na direção das linhas e colunas da imagem, como mostra a equação 10 :

$$
S_{C C D}^{\text {display }}=\left[\begin{array}{cccc}
f x & 0 & 0 & 0 \\
0 & f y & 0 & 0 \\
0 & 0 & 1 & 0
\end{array}\right]
$$

\subsection{Funcionamento do SRA}

$\mathrm{O}$ critério de determinação da área de abrangência do sistema de realidade aumentada é definido pela área da tela do smartphone e ocorre em duas etapas. A primeira etapa calcula a área de abrangência da tela do smartphone a partir das coordenadas do smartphone e dos quatro cantos da tela. Após este cálculo o sistema realiza uma busca no banco de dados local do smartphone a fim de encontrar os pontos de interesse referentes a este intervalo de coordenadas. Ao encontrar os pontos pertencentes ao intervalo os mesmos são superpostos à imagem real, através do uso das transformações anteriormente descritas é calculada a posição do elemento virtual na tela do smartphone. A segunda etapa permite a visualização dos dados com o auxílio de uma ferramenta de zoom. Esta ferramenta funciona da seguinte forma: a partir do cálculo da posição do smartphone as distâncias entre o smartphone e os pontos de interesses ou elementos virtuais selecionados na primeira etapa são calculados. Logo é possível definir até qual distância o usuário deseja ver os pontos, por exemplo, se selecionado um zoom de $2 \mathrm{~km}$, todos os elementos que 
estão a uma distância de $2 \mathrm{~km}$ serão visualizados pelo usuário, mesmo que elementos a $10 \mathrm{~km}$ também pertençam aquela área de abrangência da cena real. Esta ferramenta permite diminuir o volume de dados visualizados, a representação é restrita a uma distância definida pelo usuário, além de ser uma ferramenta opcional, que pode ser ou não utilizada pelo usuário.

O banco de dados utilizado no sistema é local, isto é, armazenado diretamente na memória do smartphone em um arquivo de pontos no formato JSON. O formato padrão aberto para JSON (JavaScript Object Notation) é compacto e baseado em um arquivo ASCII, concebido para intercâmbio de dados entre aplicações. O arquivo JSON é composto por três tipos de estruturas: um objeto que pode ser um vetor ou uma matriz com os dados e um valor. No caso dos dados censitários os objetos são os números dos setores censitários, e os valores são as respectivas coordenadas de latitude e longitude de cada setor censitário. O sistema funciona com dois arquivos JSON, um com os pontos referentes aos dados do Censo e outro com os pontos referentes aos Nomes Geográficos do Brasil. Cada arquivo possui uma lista de pontos interesse com as suas respectivas coordenadas latitude e longitude, bem como o nome que descreve o ponto e a página da web que complementa asua informação. Estes arquivos foram criados especificamente para esta aplicação e possuem apenas dez pontos de interesse em cada para a utilização nos testes. Vale resaltar que o banco de dados é consultado pelo SRA a cada mudança direção ou sentido do usuário.

Para a representação dos dados virtuais que são sobrepostos à imagem real captada pela câmara, é utilizado o pacote android.graphics que disponibiliza uma biblioteca gráfica de baixo nível, e permite a programação de ferramentas como telas, filtros de cores, pontos e retângulos para elaborar desenhos diretamente no display. A opção de utilizar primitivas gráficas simples como círculos, setas, triângulos e retângulos como objetos virtuais foi adotada para imprimir maior velocidade à representação, visto que elementos mais complexos, como texturas não foram utilizados por poder retardar a superposição dos objetos virtuais à imagem real, prejudicando a fusão.

\subsection{Representação dos dados}

A utilização de um sistema de realidade aumenta é um processo de comunição visual, no qual estão envolvidos três elementos: o desenvolvedor, o usuário do sistema e o canal de transmissão, neste caso a realidade aumentada. No módulo desenvolvido os dados estatísticos e cartográficos, Censo e Nomes Geográficos respectivamente, necessitam de variáveis visuais para a sua representação. Estes dados foram divididos em duas categorias de mesmo nome a fim de possibilitar ao usuário a visualização das categorias de forma individual ou simultaneamente.

$\mathrm{Na}$ comunicação cartográfica as variáveis visuais podem ser aplicadas em pontos, linhas e áreas. BERTIN (1983) definiu oito variáveis visuais para o plano de um mapa: duas dimensões, tamanho, valor, granulação ou textura, cor, orientação e forma. No SRA foram utilizados pontos e nestes aplicados as variáveis visuais: 
forma, tamanho, cor e orientação. As variáveis visuais textura e valor não foram utilizadas, a primeira por reduzir a velocidade de representação dos elementos virtuais e a segunda por representar hierarquia entre os objetos, porém os dados representados não possuem hierarquia entre eles.

A variável visual forma foi utilizada pois o sistema permite três tipos de representação: círculos, triângulos invertidos e setas, como mostra a Figura 2.

Figura 2 - Círculos, triângulos invertidos e setas, respectivamente.

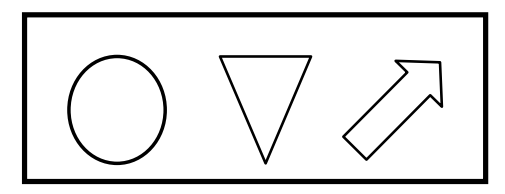

A opção de representação dos pontos de interesse com diferentes formas foi utilizada a fim de verificar qual simbologia melhor se adequa para a representação das duas categorias pesquisadas: Censo e Nome Geográfico. A variável visual tamanho foi utilizadas apenas nos pontos representados por círculos para enfatizar a distância entre o usuário e o ponto de interesse. Os pontos de interesse próximos são mostrados maiores do que os outros que estão mais distantes do usuário. A distância nos pontos representados por setas e triângulos invertidos é informada em conjunto com a descrição do objeto. A variável visual cor foi utilizada tanto para os círculos, setas quanto para os triângulos invertidos para diferenciar os pontos que são visualizados conforme a sua categoria: Censo ou Nomes Geográficos. A variável visual orientação foi utilizada apenas na representação com setas por esta opção permitir a indicação da direção dos pontos. A Tabela 4 apresenta de forma sucinta as categorias e as variáveis visuais utilizadas.

Tabela 4 - Categorias e variáveis visuais utilizadas.

\begin{tabular}{l|l|l|l|l}
\hline Categoria & $\begin{array}{l}\text { Variável } \\
\text { visual cor }\end{array}$ & $\begin{array}{l}\text { Variável } \\
\text { visual forma }\end{array}$ & $\begin{array}{l}\text { Variável } \\
\text { visual } \\
\text { tamanho }\end{array}$ & $\begin{array}{l}\text { Variável } \\
\text { visual } \\
\text { orientação }\end{array}$ \\
\hline $\begin{array}{l}\text { Nome } \\
\text { Geográfico } \\
\text { do Brasil }\end{array}$ & Azul & $\begin{array}{l}\text { Círculos, } \\
\text { triângulos } \\
\text { invertidos } \\
\text { setas. }\end{array}$ & $\begin{array}{l}\text { Possui } \\
\text { apenas na } \\
\text { opção } \\
\text { ćrculos }\end{array}$ & $\begin{array}{l}\text { Possui } \\
\text { apenas na } \\
\text { opção setas }\end{array}$ \\
\hline Censo & Vermelha & $\begin{array}{l}\text { Círculos, } \\
\text { triângulos } \\
\text { invertidos } \\
\text { setas. }\end{array}$ & $\begin{array}{l}\text { Possui } \\
\text { apenas na } \\
\text { opção } \\
\text { ćrculos }\end{array}$ & $\begin{array}{l}\text { Possui apenas na } \\
\text { opção setas }\end{array}$ \\
\hline
\end{tabular}


3.5.1 Visualização de dados

O módulo de RA desenvolvido possui quatro modos de visualização: modo de RA, modo orientação, modo lista de pontos e o modo mapa. Devido ao fato do framework permitir a sua adaptação a outras finalidades, o módulo manteve algumas funções presentes no framework como o modo de lista de pontos e o modo mapa. O modo RA foi modificado para se adaptar as categorias apresentadas nesta pesquisa. O modo orientação foi inteiramente desenvolvido. O modo mapa, apesar de ser originalmente do framework foi modificado para se adaptar as categorias utilizadas.

A seguir, os modos que formam o sistema são descritos:

Modo RA - $\mathrm{O}$ modo RA é ativado quando o usuário abre o programa, liga o GPS do smartphone e coloca o dispositivo na posição vertical (Figura 3). Nele, as categorias selecionadas são representadas como círculos (opção padrão) ou como triângulos invertidos. As representações são inseridas na posição correspondente ao ponto de intersse. Juntamente com o ponto segue uma pequena descrição do mesmo. Este modo apresenta a opção zoom anteriormente explicado no item 3.4 Funcionamento do SRA.

Figura 3 - Exemplo de um smartphone na posição vertical e sua respectiva visualização.
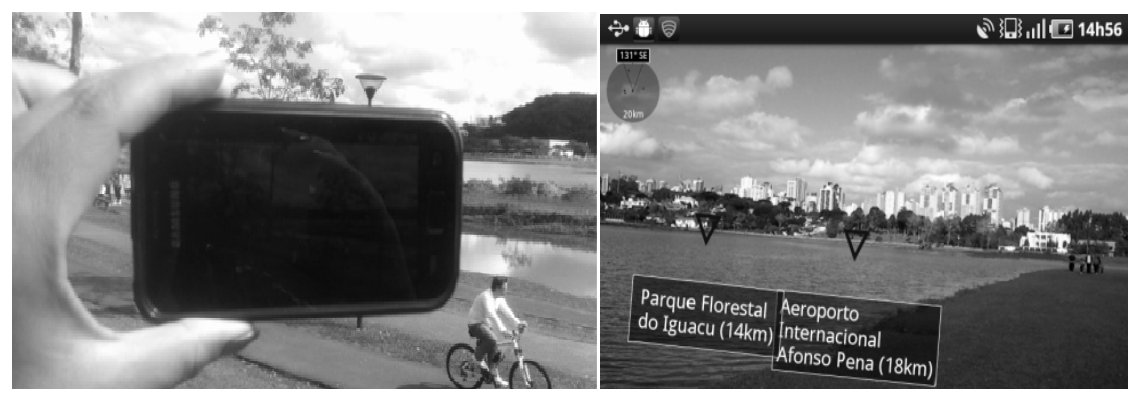

Modo orientação - Quando o usuário coloca o dispositivo na posição horizontal (Figura 4), é possível selecionar no menu a opção setas e os pontos de interesse são apresentados como setas mediante a seleção desta opção. As setas indicam a direção de cada ponto, a distância e o nome do elemento. Esta opção foi gerada para facilitar a locomoção do usuário sem a necessidade de manter o smartphone na posição vertical. 
Figura 4 - Exemplo de um smartphone na posição horizontal e sua respectiva visualização.

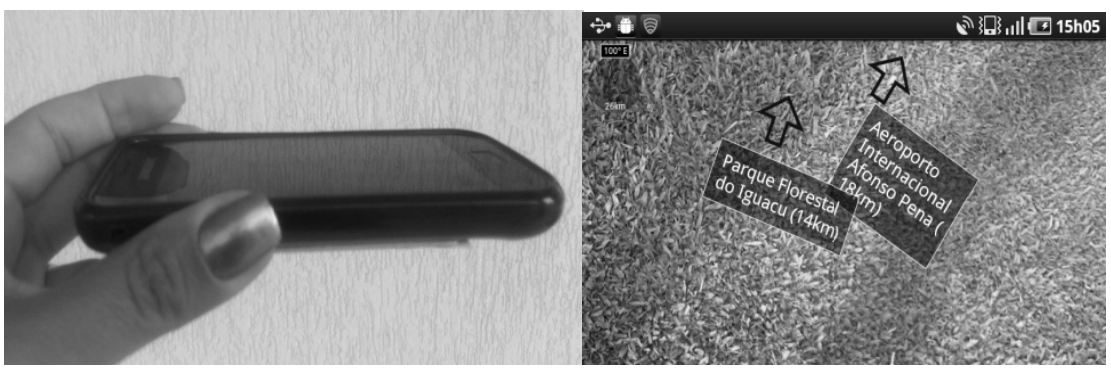

Estes dois modos de visualização permitem também ao usuário selecionar um objeto mediante um toque em um dos pontos na tela do dispositivo. Assim, o sistema abre uma página HTML com maiores informações previamente selecionadas sobre o ponto. As páginas HTML podem ser relativas a páginas previamente existentes ou a uma página personalizada criada especificamente para $\mathrm{o}$ ponto de interesse, como no caso desta pesquisa. A Figura 5 mostra o ponto referente ao Setor 32 e as informações sobre este setor.

Figura 5 -Página html sobre o setor 32 selecionado na tela do smartphone.

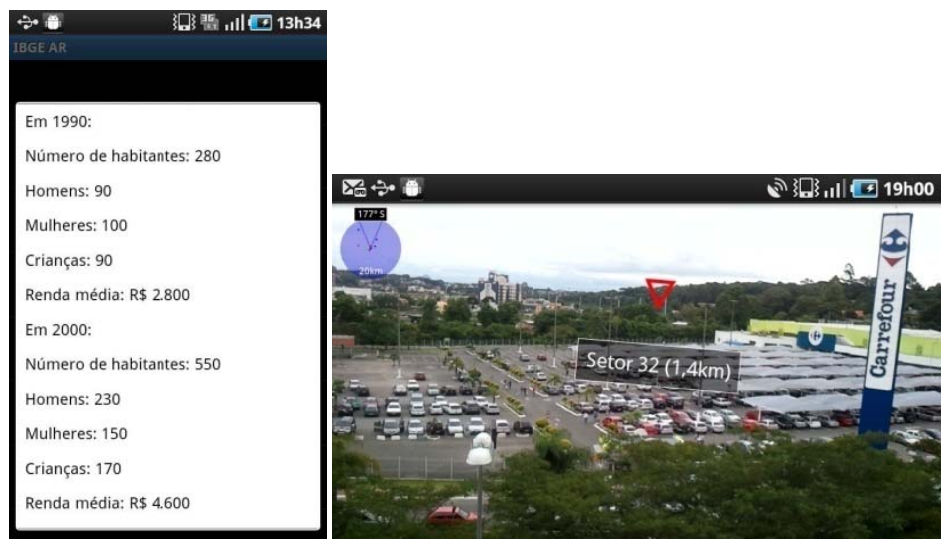

Através destas páginas é possível visualizar outras informações como gráficos e estatísticas da região. O acesso a página da web pode ser usado de forma independente, o usuário do programa pode abrir links a partir da página principal, fazer um registro ou deixar um comentário, por exemplo. Então, o usuário pode retornar ao programa de RA. Os outros dois modos do programa são autoexplicáveis como segue.

Bol. Ciênc. Geod., sec. Artigos, Curitiba, v. 18, noํ, p.282-301, abr-jun, 2012. 
Modo lista de pontos - apresenta os pontos das respectivas categorias selecionadas como uma lista de nomes, ordenados a partir da menor distância do ponto em relação a posição do smartphone. Este modo também permite o acesso à respectiva página da Internet.

Modo mapa - exibe uma visão geral dos pontos das respectivas categorias selecionadas e a posição do usuário representados em um mapa através da utilização do Google Maps. A função MapView requer uma conexão de Internet permanente para funcionar corretamente, pois os dados são recebidos diretamente do servidor de Google APIs. Assim, o sistema exibe automaticamente o mapa em 2D (Google Map) com a posição do usuário indicada e é possível interagir com os mapas, com a opção de zoom e visualizar os pontos de interesse num contexto de fronteiras.

\section{RESULTADOS}

Alguns dos resultados obtidos tanto para os dados do censo como para os nomes geográficos são descritos a seguir. Para a área de estudo foi utilizado o município de Curitiba/PR, especificamente o bairro do Campo Comprido.

Inicialmente deve ser selecionada a categoria que deseja ser visualizada, por exemplo, "Censo" ou "Nomes Geográficos". Os pontos de interesse pertencentes a cada categoria podem ser visualizados de forma conjunta através da seleção de ambas as categorias ou de forma individual. Os elementos pertencentes às categorias selecionadas são visualizados e diferenciados através da variável visual cor.

A Figura 6 apresenta o SRA no modo orientação, nesta opção a representação inclui setas na imagem e através de sua direção mostram a posição relativa dos pontos pertencentes à categoria Nomes Geográficos, o que permite ao usuário a navegação e consulta dos pontos de forma simultânea.

Figura 6 - Variável visual cor, forma e orientação.

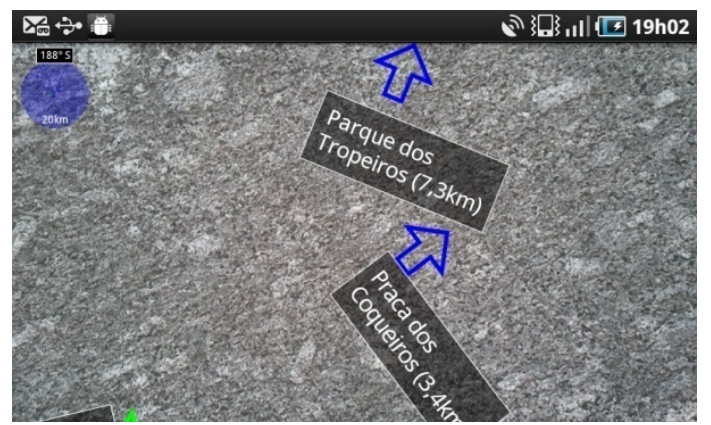

No modo RA, quando o telefone está na posição vertical, os objetos são descritos por meio de sua posição, nome e distância ao usuário. A separação das 
diferentes categorias se realiza pela aplicação da variável visual cor como mostra a Figura 7. Sendo a cor vermelha utilizada para a categoria Censo e azul para a categoria Nomes Geográficos.

Figura 7 - Variável visual cor e forma.

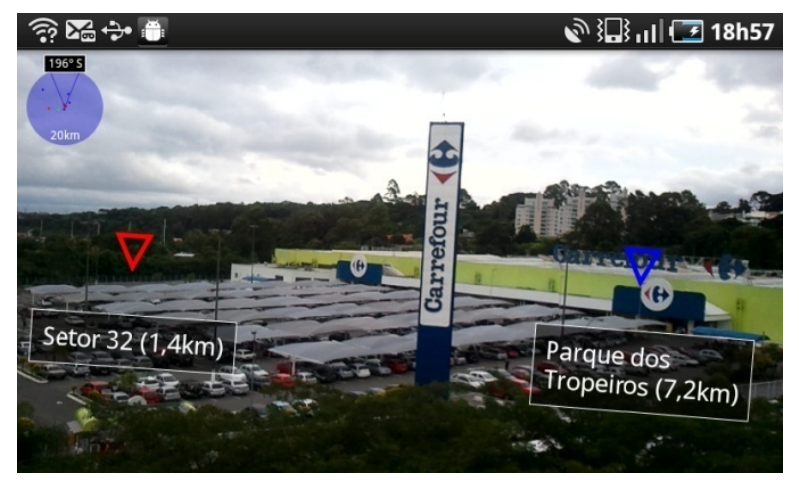

Já a distância pode ser enfatizada com a aplicação da variável visual tamanho e a forma círculo, como mostra a Figura 8. Quanto mais próximo o ponto está do usuário maior o tamanho do círculo.

Figura 8 - Variável visual cor, tamanho e forma.

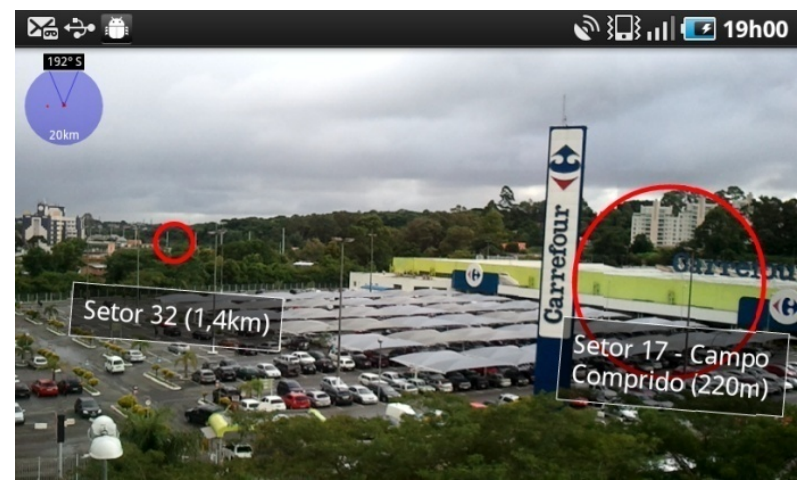

Com a finalidade de validar a variável visual forma, duas opções foram verificadas. A primeira é o uso de círculos, como mostrado anteriormente. A segunda é a substituição dos círculos por triângulos invertidos. A Figura 9 apresenta o mesmo ponto Parque Iguaçu pertencente à categoria nome geográfico em azul, representada por triângulos invertidos na Figura 9a e por círculos na Figura 9b. Esta 
figura permite uma comparação visual na visualização do mesmo ponto com dois símbolos distintos. Nota-se que ambos os símbolos, apesar de construídos de forma distinta, localizam o ponto na mesma região o que indica o funcionamento correto do SRA, independente do símbolo escolhido.

Figura 9 - RA com triângulos invertidos (a) e círculos (b): variável visual forma.

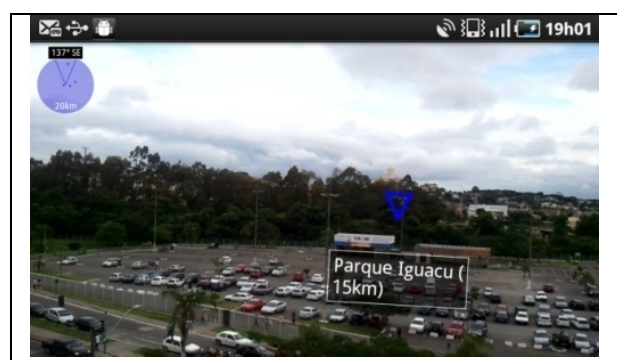

(a)

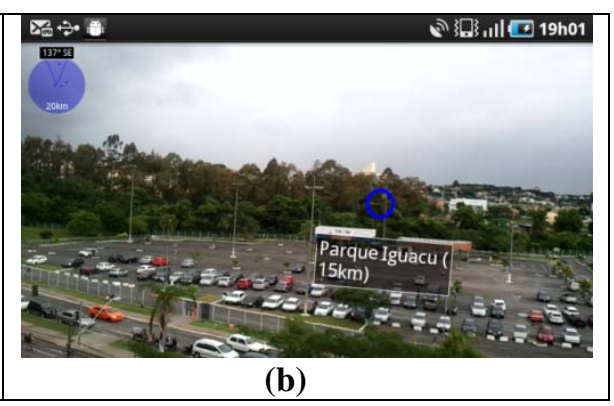

(b)

Como o tamanho da tela é restrito, não é recomendado incluir muitas informações no display. Por isso, para a obtenção de outras informações sobre o ponto de interesse foram geradas webpages personalizadas para cada ponto, as quais podem ser acessadas através do toque na tela sensível. A associação da webpage desejada ao ponto é indicada no banco de dados local que contem as informações referentes às coordenadas dos pontos.

\section{DISCUSSÕES E CONCLUSÃO}

A visualização de dados estatísticos e cartográficos produzidos e divulgados pelo IBGE poderá no futuro ser realizada a partir de um SRA, a pesquisa apresentada mostra apenas um pequeno passo nesta direção. Estes dados podem auxiliar e simplificar a interpretação do usuário de uma área de estudo. Tal área poderá ser estudada através do SRA visando diversas futuras aplicações como a realização de novas pesquisas, implantação de obras, projetos de desenvolvimento de uma região, facilitação de trabalhos de campo em áreas extensas, soluções de problemas de localização de um determinado elemento em campo, entre outros. $\mathrm{O}$ SRA poderá no futuro permitir um maior conhecimento do território nacional, bem como dos elementos presentes no mapeamento. Para isso é necessário um amplo estudo sobre a linguagem cartográfica a ser utilizada, pois esta depende da informação que está sendo representada e do contexto no qual os símbolos aparecerão no SRA. A simplificação da visualização dos dados, principalmente com a apresentação de pequenos resumos em páginas web especificamente desenvolvidas, poderá atrair novos usuários que antes consideravam tais informações irrelevantes ou complexas para a sua interpretação. 
A possibilidade de desenvolvimento de SRA para smartphones apresenta diversas vantagens dentre elas o fato do usuário possuir completa mobilidade quando estiver utilizando o sistema. Outro fator a ser salientado é que o SRA não requer nenhuma infraestrutura adicional ou outros hardwares de alto custo e apresentou-se nos testes de forma prática e eficiente. No desenvolvimento de um SRA para Android apenas softwares livres são utilizados. O banco de dados empregado pode ser atualizado no smartphone de modo que apenas uma parte do banco de dados reside no telefone, pois este permite armazenar apenas a região de interesse.

Os dispositivos de determinação de posição e atitude não oferecem alta acurácia, mas isto não restringe o uso destes equipamentos. As aplicações devem considerar esta limitação. No caso da visualização de dados do IBGE, a exigência de acurácia não é alta, pois a intenção é enriquecer a cena com informações sobre objetos localizados a distância. É esperado que num futuro próximo este tipo de aplicações receba maior atenção dentro da Cartografia, como consequência da popularização de dispositivos móveis como os tablets.

Para que o sistema possa se comunicar de maneira eficiente a informação que está sendo transmitida é necessário considerar todas as condições que influenciam este processo, ou seja pesquisas com usuário para estabelecer suas reais necessidades, bem como pesquisas sobre as variáveis visuais adequadas para cada caso, além do estudo da possibilidade de aplicação da simbologia cartográfica no SRA.

\section{AGRADECIMENTOS:}

O autor gostaria de agradecer a CAPES (Processo n. 0074/10-1) pelo o apoio financeiro dado a esta pesquisa. O autor também agradece a UFPR, ao IBGE e ao Karlsruher Institut für Technologie - KIT pelo apoio dado a este trabalho.

\section{REFERÊNCIAS BIBLIOGRÁFICAS}

AZUMA, R.; et al. Recent Advances in Augmented Reality. IEEE Computer Graphics and Applications, v .21, n.6, November - December 2001, p. 34-47. Disponível em: < http://www.cs.unc.edu/ azuma/cga2001.pdf>. Acesso em: 26 de janeiro de 2011.

BERTIN, J. Semiology of Graphics, Diagrams, Networks, Maps. The University of Wisconsin Press, London, 1983, 415 p.

DOMHAN, T. Augmented Reality on Android Smartphones. Des Studiengangs Informationstechnik an der Dualen Hochschule Baden-Württemberg Stuttgart, 8. Juni, 2010.

GARTNER GROUP. Disponível em: <http://www.gartner.com/technology/ home.jsp>. Acesso em: 05 de julho de 2011.

GASSMANN, A. GuidAce - Augmented Reality on Android, Server-side recognition and client-side tracking. Dissertação de Mestrado. Swiss Federal 
Institute of Technology Zurich. Department of Information Technology and Electrical Engineering. 2010.

GONZAlEZ, R.; WOODS, R. Digital Image Processing, 3rd Edition, Prentice HALL, 2007, 976 p.

IBGE. Glossário dos termos genéricos dos nomes geográficos utilizados no mapeamento sistemático do Brasil - vol. 1 - Escala 1:1.000.000. 2011.

MAQABLEH, W. F., SIDHU, M. S.: From boards to augmented reality learning. International Conference on Information Retrieval \& Knowledge Management (CAMP), pp. 184-187, 17-18 March 2010.

MARIMON D.; et.al. MobiAR: Tourist Experiences through Mobile Augmented Reality. Telefonica Research and Development, Barcelona, Spain; Visual Interaction Communication Technologies Vicomtech-IK4, San Sebastian, Spain; LabHuman, Valencia, Spain, Indra Software Labs, Madrid, Spain, Brainstorm, Valencia, Spain; 2010.

MEIER, R. Professional Android 2 Application Development. Wiley India Pvt. Ltd., April 2010, $576 \mathrm{p}$.

MILGRAM, P.; KISHINO, F. A Taxonomy of Mixed Reality Virtual Displays. IEICE Transactions on Information and Systems, E77-D, 9, September 1994, p. 1321-1329. Disponível em: <http://web.cs.wpi.edu/ gogo/hive/ papers/Milgram_IEICE_1994.pdf>. Acesso em: 02 de fevereiro de 2011.

MIYASHITA, T. ; et. al. An Augmented Reality museum guide. In: ISMAR 2008 Proceedings of the 7th IEEE/ACM International Symposium on Mixed and Augmented Reality, IEEE Computer Society Washington, DC, USA, 2008, p. 103 - 106. Disponível em: <http://ieeexplore.ieee.org/xpls/abs_all.jsp? arnumber $=4637334 \& \operatorname{tag}=1>$. Acesso em: 02 de fevereiro de 2011.

RADBURN, A. A Mobile Augmented Reality Demonstrator. 2006. Disponível em: $<$ http://regard.crg.ulaval.ca/2006/proceedings/09-radburn.pdf $>$. Acesso em 28 de setembro de 2011.

SUTHERLAND, I., A head-mounted three dimensional display. In: Proc. FJCC 1968, pages 757-764, Washington, DC. Thompson Books, 1968.

WENG, E. N. G.; et. al. Mobile phone augmented reality business card. International Journal of Computer Science and Information Security, v. 9, Issue: 5, 2011, p. 154-164.

(Recebido em fevereiro de 2011. Aceito em maio de 2012.) 